\title{
The Far Future of Exoplanet Direct Characterization
}

\author{
Jean Schneider, Alain Léger, ${ }^{2}$ Malcolm Fridlund, ${ }^{3}$ Glenn J. White, ${ }^{4,5}$ Carlos Eiroa, ${ }^{6}$ Thomas Henning, \\ Tom Herbst, ${ }^{7}$ Helmut Lammer, ${ }^{8}$ René Liseau, ${ }^{9}$ Francesco Paresce, ${ }^{10}$ Alan Penny, ${ }^{5,11}$ Andreas Quirrenbach, ${ }^{12}$ \\ Huub Röttgering, ${ }^{13}$ Franck Selsis, ${ }^{14}$ Charles Beichman, ${ }^{15}$ William Danchi, ${ }^{16}$ Lisa Kaltenegger, ${ }^{17}$ \\ Jonathan Lunine, ${ }^{18}$ Daphne Stam, ${ }^{19}$ and Giovanna Tinetti ${ }^{20}$
}

\begin{abstract}
We describe future steps in the direct characterization of habitable exoplanets subsequent to medium and large mission projects currently underway and investigate the benefits of spectroscopic and direct imaging approaches. We show that, after third- and fourth-generation missions have been conducted over the course of the next 100 years, a significant amount of time will lapse before we will have the capability to observe directly the morphology of extrasolar organisms. Key Words: Far future missions-Direct imaging-High-resolution spectroscopy-Habitable exoplanets-Exo-moons—Surface features. Astrobiology 10, 121-126.
\end{abstract}

\section{Investigating More Exoplanets or Habitats}

$\mathbf{T}$ HE FUTURE OF EXOPLANETOLOGY is a priori bright since we already know that at least $30 \%$ of main sequence stars have one or several super-Earth companions (Mayor et al., 2009). It is likely that there will be two generations of space missions for the direct characterization of exoplanets in the next $15-25$ years:

$>$ a first generation with a $1.5-2 \mathrm{~m}$ class coronagraph suited for giant planets and nearby super Earths (e.g., Schneider et al., 2009)

followed by

a second generation consisting of an interferometer (Lawson et al., 2008; Cockell et al., 2009), an external occulter (Glassman et al., 2009), a large $8 \mathrm{~m}$ class coronagraph (visible; Shaklan and Levine, 2008), a Fresnel interferometric imager (Koechlin et al., 2009) or a $20 \mathrm{~m}$ segmented coronagraph (super James Webb Space Telescope) for the near IR (Lillie et al., 2001).

In parallel, there will likely be coronagraphic cameras on ground-based extremely large telescopes (like the EPICS camera; Kasper et al., 2008). Here, we have attempted to anticipate what should come next and consider a thirdgeneration mission along with future implications.

There are essentially two directions for astronomers to pursue: (1) the investigation of a larger volume of planets and (2) broader, more detailed characterization of exoplanets for which a candidate biomarker* has been found, to

\footnotetext{
${ }^{1}$ Observatoire de Paris-Meudon, Laboratoire de l'Univers et ses Théories, Meudon, France.

${ }^{2}$ Institut d'Astrophysique Spatiale, Université Paris-Sud, Orsay, France.

${ }^{3}$ Research and Scientific Support Department, ESA, European Space Research and Technology Centre, Noordwijk, the Netherlands.

${ }^{4}$ The Open University, Milton Keynes, UK.

${ }^{5}$ Space Science \& Technology Department, CCLRC Rutherford Appleton Laboratory, Oxfordshire, UK.

${ }^{6}$ Universidad Autonoma de Madrid, Madrid, Spain.

${ }^{7}$ Max-Planck Institut für Astronomie, Heidelberg, Germany.

${ }^{8}$ Space Research Institute, Austrian Academy of Sciences, Graz, Austria.

${ }^{9}$ Department of Radio and Space Science, Chalmers University of Technology, Onsala, Sweden.

${ }^{10}$ Istituto Nazionale di Astrofisica, Rome, Italy.

${ }^{11}$ Royal Observatory Edinburgh, Blackford Hill, Scotland.

${ }^{12}$ Landessternwarte, Heidelberg, Germany.

${ }^{13}$ Leiden Observatory, Leiden, the Netherlands.

${ }^{14}$ University of Bordeaux 1, Bordeaux, France.

${ }^{15}$ NASA Exoplanet Science Institute, California Institute of Technology and Jet Propulsion Laboratory, Pasadena, California, USA.

${ }^{16}$ NASA Goddard Space Flight Center, Greenbelt, Maryland, USA.

${ }^{17}$ Harvard-Smithsonian Center for Astrophysics, Cambridge, Massachusetts, USA.

${ }^{18}$ Lunar and Planetary Laboratory, Tucson, Arizona, USA.

${ }^{19} \mathrm{SRON}$, Netherlands Institute for Space Research, Utrecht, the Netherlands.

${ }^{20}$ Department of Physics and Astronomy, University College London, London, UK.

*The term biomarker is used here to mean detectable atmospheric species or set of species whose presence at significant abundance strongly suggests a biological origin.
} 
include securing biomarkers as well. We address these possibilities from a scientific point of view rather than one of mission design. We have limited our study to planets whose radius is larger than 0.5 that of Earth, though smaller planets were considered as habitats but may not have evolved in the manner of an Earth-type habitable planet (Lammer et al., 2009).

The detection of yet-undiscovered exoplanets that are the same distance from Earth as those detected by the first two generations of missions will not require a newly devised mission design. To detect a larger number of habitable exoplanets, it will be necessary to observe more-distant stars (hereafter "more distant" means farther than $50 \mathrm{pc}$ ), or telluric-like moons of known giant planets in the habitable zone of their nearby star (hereafter "nearby" means closer than 20 pc). In both cases, an increase in angular resolution will be required, either to separate distant planets from their parent star or to separate a telluric companion from its parent giant planet. At $50 \mathrm{pc}$, the baseline required to separate a planet at $1 \mathrm{AU}$ from the star is $B=12 \mathrm{~m}$ at $600 \mathrm{~nm}^{1}$ (respectively $B=200 \mathrm{~m}$ at 10 micron). But, for distant planets, angular resolution is not sufficient. The collecting area must also scale as $D^{2}$ where $D$ is the distance of the planetary system. For a single aperture, this condition is automatically fulfilled; for an interferometer, it is an extra constraint.

\section{Deeper Characterization of Most-Interesting Planets}

\subsection{Spectroscopic and polarimetric approach}

One can search for weaker lines (like $\mathrm{CO}_{2}$ around 9.3 and 10.5 micron in the thermal emission spectrum; Fig. 1), for narrower lines, and for the detailed spectral line shape thanks to higher spectral resolution. The latter case is well illustrated by the double peak of the $\mathrm{O}_{2}$ band at $760 \mathrm{~nm}$ and of $\mathrm{O}_{3}$ at 9.6 micron, and the $\mathrm{CO}_{2}$ central peak at 15 micron (Fig. 1). The $\mathrm{CO}_{2}$ central peak is an interesting diagnostic of temperature inversion in the planetary atmosphere: the central structure of the $\mathrm{CO}_{2}$ band informs that the upper atmosphere is warmer than the mid-altitude regions, which indicates the presence of an absorbing gas in the former region. ${ }^{2}$ Spectroscopy of giant planets with sufficient signal-tonoise ratios $(\mathrm{S} / \mathrm{N})$ and spectral resolution will also allow for measurement of their radial velocity. This will incidentally lead to the determination of the mass of their parent star with an unprecedented accuracy and will allow, by measuring the period and amplitude of the radial velocity variation, detection of moons and measurement of their mass and distance from the host planet. Indeed, the amplitude of the planetary radial velocity variation for a moon at a distance

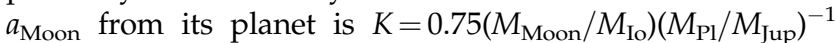
$\left(a_{\text {Moon }} / a_{\mathrm{IO}}\right)^{-1 / 2} \mathrm{~km} \mathrm{~s}^{-1}$. The polarimetric approach will improve the knowledge of clouds, surface, rings, and so forth. (Stam, 2008).

\footnotetext{
${ }^{1}$ We assume that planets must be at an angular distance larger than $2 \lambda / B$ to be observable by a coronagraph.

${ }^{2}$ For a strongly absorbing gas, the intensity of the emission, at a given wavelength, is essentially that of a blackbody at the temperature of the atmosphere at the altitude where the optical depth $\tau$ from outside the background is unity. Consequently, the emission at the center of a band comes from regions at higher altitude than that in the band's wings, revealing the temperature of these regions.
}

\subsection{Direct imaging approach}

By progressively increasing the angular resolution, the following can be achieved:

$>$ Detect habitable moons of giant planets by separating the moon's image from that of its parent planet. To separate a moon that is $0.003 \mathrm{AU}$ (the Io-Jupiter distance) from its parent giant planet at $10 \mathrm{AU}$ requires a baseline $B=400 \mathrm{~m}$ at $600 \mathrm{~nm}$ (respectively $B=7 \mathrm{~km}$ at 10 micron).

$>$ Improve the transit spectroscopy of transiting planets (Schneider, 2000). With a baseline $B=645 \mathrm{~m}$ at $600 \mathrm{~nm}$, a pixel with a size $=0.1$ solar radii $\left(R_{\text {Sun }}\right)$ on a star at $50 \mathrm{pc}$ can be isolated (until now there are only six transiting planets closer than $50 \mathrm{pc}$ ); therefore, the $\mathrm{S} / \mathrm{N}$ can be improved by a factor 10 . Note that, for this case, no high-contrast imaging is required; this would be an excellent application of the Stellar Imager project (Carpenter et al., 2009).

> Perform astrometric detection of moons. The astrometric measurement of the displacement of the planet's position due to the pull by a moon offers another way to detect companions to planets. The required baseline is $B=150,000 \quad(D / 10 \mathrm{pc})\left(a_{\mathrm{Moon}} / a_{\mathrm{Io}}\right)^{-1}\left(M_{\mathrm{Pl}} / M_{\mathrm{Jup}}\right)$ $\left(M_{\text {moon }} / M_{\mathrm{Io}}\right)^{-1} \mathrm{~km}$ at $600 \mathrm{~nm}$.

$>$ Constrain the planetary radius for transiting planets. The accurate astrometric measurement of the star's centroid during the transit of a planet will give a measurement of the planetary radius independent from the photometry of transits. Indeed, the position of the centroid varies during the transit with a linear amplitude $R_{\mathrm{Pl}}^{3} / R_{\mathrm{Star}}^{2}$, which corresponds to a few microarseconds for a Jupiter-sized planet at 10 pc. (Schneider, 2000).

> If an isolated ("free floating") planet is accompanied by a low-mass companion ("moon"), the observation of its orbit will be used to infer the mass of the parent planet.

$>$ Direct measurement of the planetary radius: knowledge of the planetary radius is important since this parameter controls the surface gravity and the Jeans escape of molecules. It can be inferred indirectly from the transit depth (for the few transiting planets) and constrained, with the help of atmosphere models, from the planetary flux in reflected light and thermal emission. A direct measurement is obtained with an imager that has an angular resolution of, say, $0.3 R_{\mathrm{Pl}}$. For a $2 R_{\text {Earth }}$ planet at $5 \mathrm{pc}$, the required baseline at $600 \mathrm{~nm}$ is $B=20 \mathrm{~km}$.

The ultimate step, currently, would be the direct imaging of surface features (oceans, continents). In this configuration, astronomers can search for the direct detection of the ocean's glint (Williams and Gaidos, 2008). This approach is particularly interesting for imaging forests and savannahs in order to investigate at a moderate spectral resolution the equivalent of the "red edge" of terrestrial vegetation at $725 \mathrm{~nm}$. To have, for example, a $10 \times 10$ pixel image of a $2 R_{\text {Earth }}$ planet at $5 \mathrm{pc}$, a baseline $B=70 \mathrm{~km}$ is required at $600 \mathrm{~nm}$.

These different configurations are summarized in Table 1 for an image at $600 \mathrm{~nm}$. For an image at 10 micron, the required baseline is multiplied by 17 . But, for direct imaging, the angular resolution is not sufficient. A sufficiently large collecting area is also necessary, which adds an additional 


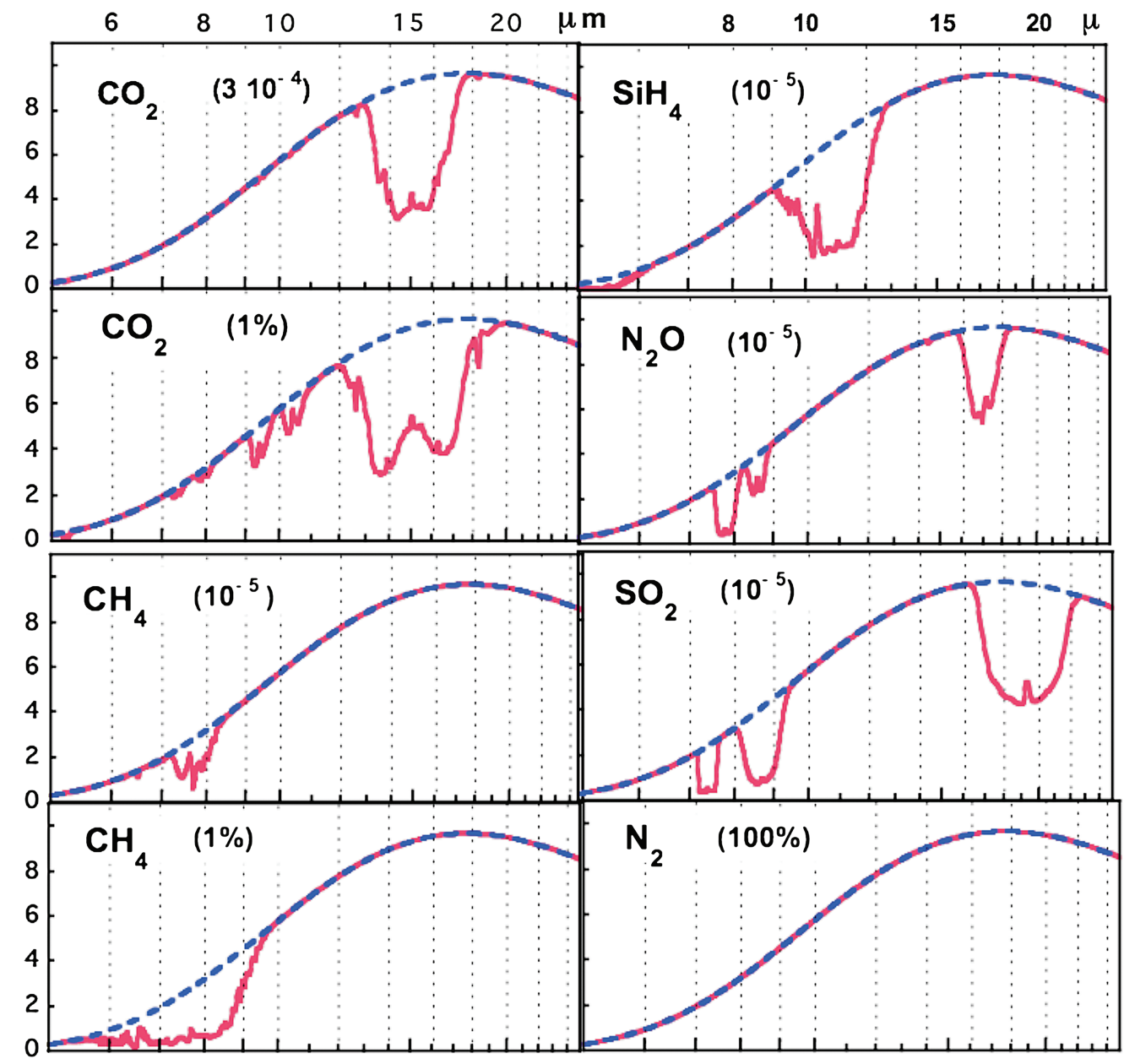

FIG. 1. Spectra of the thermal emission of planets somewhat similar to Earth but with different gases in their atmospheres. The spectral resolution is $\mu \lambda / \Delta \lambda=200$ and $\mathrm{S} / \mathrm{N} \sim 100 \sigma$. An additional non-IR-active gas, for example, $\mathrm{N}_{2}$ or $\mathrm{O}_{2}$, is assumed with a pressure of 1 bar. The thermal profile of the atmosphere is that of Earth. All the spectral features present are real and show how precise are the fingerprints of gases at this spectral resolution (about 10 times larger than that of Darwin in the present version). In particular, an estimate of the abundance of the gases is possible. The reader is invited to compare the cases of $\mathrm{CO}_{2}$ and $\mathrm{CH}_{4}$ at different concentrations (with respect to the 1 bar of inert gas). As explained in the text, the central structure of strong bands, for example, $\mathrm{CO}_{2}$, are informative about the thermal structure of the atmosphere (warm stratosphere). Color images available online at www.liebertonline.com/ast.

constraint on sparse aperture interferometers like the "hypertelescope" (Labeyrie, 1996). To have the same S/N as that for a single pixel image of a planet with a $2 \mathrm{~m}$ (respectively $8 \mathrm{~m}$ ) single aperture, a total area equivalent to a single aperture of $20 \mathrm{~m}$, or 900 one-meter apertures, (respectively $80 \mathrm{~m}$, or 6400

Table 1. Baseline Distances Necessary for Exo-Moon INVESTIgATIONS TO Direct IMAgING OF Exoplanetary Surface Features (after Williams and Gaidos, 2008)

\section{Objectives}

Exo-moon image

Spectroscopy of a planetary transit image

Astrometry of a planetary transit image

Direct measurement of planetary size

Image of continents/oceans
Required baseline at $600 \mathrm{~nm}$

$400 \mathrm{~m}$

$645 \mathrm{~m}$

$40 \mathrm{~km}$

$20 \mathrm{~km}$

$70 \mathrm{~km}$ one-meter apertures) is required to have a $10 \times 10$ pixel image of a planet.

In conclusion, with a few exceptions, large baselines will be required in the future to perform direct imaging and, in some cases, spectroscopic observations of exoplanets. Therefore, astronomers will inevitably be led to design large interferometers, even at short visible wavelengths. An intermediate step on this pathway would be a mission like the Stellar Imager (Carpenter et al., 2009), for which no additional highcontrast imaging performances are required.

\section{Other Studies}

\subsection{Long-term monitoring of most-interesting planets}

A better knowledge of behavior and characteristics of the most-interesting planets will be provided by long-term monitoring programs over the course of months to years. These programs will lead to an improved knowledge of these planets' diurnal rotations, random cloud coverage, and 
seasonal and volcanic events. A particular application is the detection of moons by mutual events during a continuous photometric monitoring of the planetary flux. These mutual events will reveal the presence of a moon by the shadow they project on the planet, by their disappearance in the planet's shadow, and by the primary and secondary transit with the parent planet (Cabrera and Schneider, 2007).

\section{2. "Technosignatures"}

Beyond standard biosignatures, another type of signal far from equilibrium can be seen as technosignatures, that is, spectral features that cannot be explained by complex organic chemistry, such as laser emissions. In the present state of our knowledge, we cannot eliminate these signals a priori, though we have no guiding lines with which to search for them. For instance, in the present Earth atmosphere, chlorofluorocarbon (CFC) gases are the result of technological chemical synthesis. Observed over interstellar distances, they would reveal to the observer the presence of technology on our planet. The detection of their absorption spectrum on an exoplanet would require a spectral resolution of at least 100 around 10 micron (See Fig. 2). Of course, in an exoplanet, CFCs would probably be replaced by other technosignatures. Another approach would be to detect artificially produced light (e.g., laser light). On Earth, the present total energy production is about $40 \mathrm{TW}$. This represents one thousandth of the sunlight energy reflected by the whole Earth. With regard to an exoplanet, this means that artificial light produced with the same power would be lost in the background noise of the stellar light reflected by the planet. This situation can be circumvented by observing the planet in the nightside only. But then the spatial resolution should be at least $0.3 R_{\mathrm{Pl}}$, which corresponds to a baseline $B$ of $70 \mathrm{~km}$ for a $2 R_{\text {Earth }}$ planet at 5 pc. To be detected with a $S / N$ equivalent to the detection of the reflected stellar light by the whole planet with a $1.5 \mathrm{~m}$ telescope, the collecting area required to detect artificial light one million times fainter should be one million times larger, that is, correspond to a single aperture with a diameter $B=1.5 \mathrm{~km}$. Another type of technosignature could go beyond the suggestion to detect artificial constructions by their transits in front of stars (Arnold, 2005); this kind of detection would be improved by resolving the stellar disc.

\section{The Very Long-Term Perspective}

If around 2020-2030 we have found a promising biomarker candidate on a nearby planet [for instance, around Alpha Centauri (Guedes et al., 2008)], such a discovery would trigger two kinds of projects:

$>$ Direct visualization of living organisms. To detect directly the shape of an organism 10 meters in length and width, a spatial resolution of 1 meter would be required. Even on the putative closest exoplanet, Alpha Centauri $\mathrm{A} / \mathrm{B} \mathrm{b}$, the required baseline would be at $600 \mathrm{~nm} B=600,000 \mathrm{~km}$ (almost the Sun's radius). In reflected light, the required collecting area to obtain 1 photon per year in reflected light is equivalent to a single aperture of $B=100 \mathrm{~km}$. In addition, if this organism is moving with a speed of $1 \mathrm{~cm} \mathrm{~s}^{-1}$, it would have to be detected in less than $1000 \mathrm{~s}$. To get a detection in 20 minutes with a $\mathrm{S} / \mathrm{N}$ of 5 , the collecting area would correspond to an aperture $B=3$ million $\mathrm{km}$. All these numbers are unrealistic, unless laser-trapped mirrors, as proposed by Labeyrie et al. (2005), are realized (in their present conception, laser-trapped mirrors are fragile in the solar wind).

$>$ Exploration of nearby stars. The possibility to explore in situ nearby stars at a speed of $0.3 \mathrm{c}$ has often been invoked (see, for instance, Crawford, 1990; Bjørk, 2007). At this speed, however, the problem of shielding against damaging cosmic rays and interstellar dust would threaten the entire mission. According to Semyonov (2009), a water shell of $1 \mathrm{~m}$ in thickness would be sufficient protection, but then there is the problem of accelerating an object up to $0.3 \mathrm{c}$. As for the threat posed by interstellar dust, a 100 micron interstellar grain at $0.3 \mathrm{c}$ would have the same kinetic energy as a 100 ton body at $100 \mathrm{~km} \mathrm{~h}^{-1}$. No currently available technology could protect against such a threat without a spacecraft that has a mass of hundreds of tons, which, in turn,

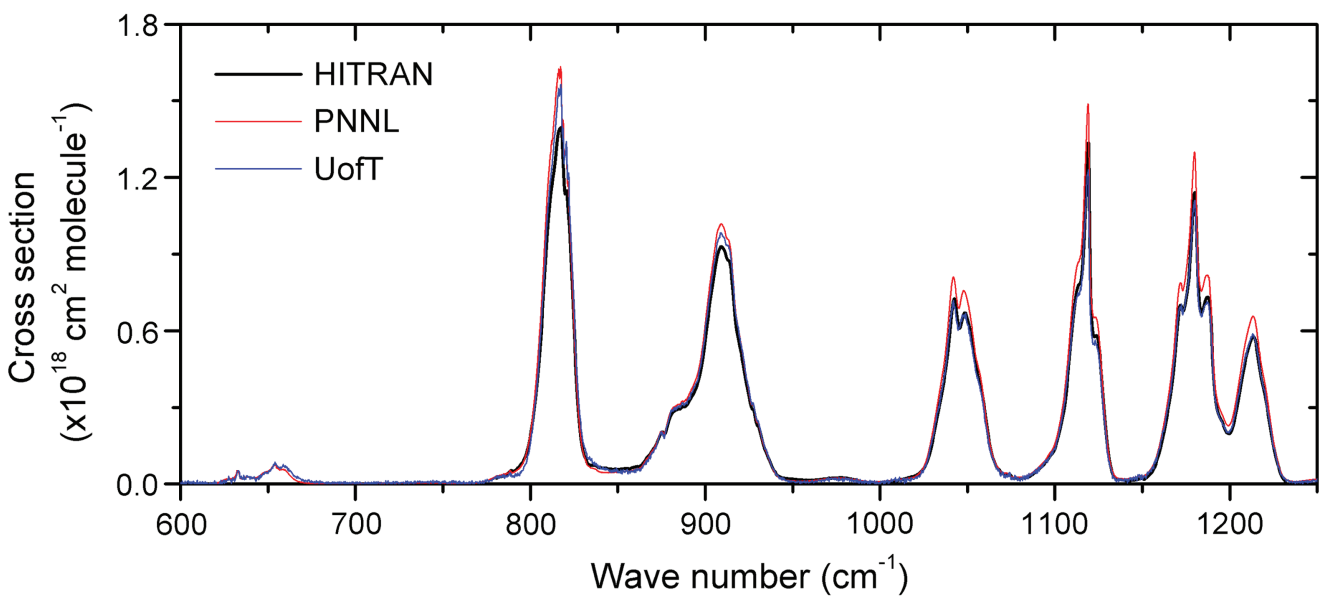

FIG. 2. Spectrum of CFC-113 (Le Bris et al., 2006), showing good agreement between the HITRAN model and Pacific Northwest National Laboratory (PNNL) and University of Toronto (UofT) measurements. Color images available online at www.liebertonline.com/ast. 
would be extremely difficult to accelerate up to $0.3 \mathrm{c}$. A way around all this would be to employ a travel velocity of only a few hundred $\mathrm{km} \mathrm{s}^{-1}$, as is the case for the "The Project" (Kilston, 1999). But then the journey to Alpha Centauri would take 10,000 years.

Regardless of the approach, it seems impossible to have direct visual contact with living organisms on a nearby exoplanet over the course of centuries, at least in the framework of foreseeable physical and technological concepts, and what physics will be in 1000 years is not reasonable to anticipate. We are thus limited by a kind of conceptual or knowledge horizon.

\section{Conclusion}

Within a period of approximately 200 years, it can reasonably be expected that high-resolution spectroscopy and then high-angular direct imaging will improve considerably our knowledge of nearby exoplanets and possible global biomarkers. For the latter approach, large interferometers will be inevitable. The highly desirable next step would be direct visual observation of the morphology of life-forms on these planets, should such life-forms exist. Unfortunately, technological obstacles will lead to a frustrating period of many centuries before this hope can be realized, and we are perhaps as far from this epoch as Epicurus was from seeing the first other worlds when, 23 centuries ago, he predicted the existence of these planets (Epicurus, 300 BCE).

\section{Abbreviations}

CFCs, chlorofluorocarbons; S/N, signal-to-noise ratio.

\section{References}

Arnold, L. (2005) Transit lightcurve signatures of artificial objects. Astrophys. J. 627:534-539.

Bjørk, R. (2007) Exploring the Galaxy using space probes. Int. J. Astrobiology 6:89-93.

Cabrera, J. and Schneider, J. (2007) Detecting companions to extrasolar planets using mutual events. Astron. Astrophys. 464:1133-1138.

Carpenter, K., Schrijver, C., and Karovska, M. (2009) The Stellar Imager (SI) project: a deep space UV/optical interferometer to observe the Universe at 0.1 milli-arcsec angular resolution. Astrophys. Space Sci. 320:217-223.

Cockell, C.S., Herbst, T., Léger, A., Absil, O., Beichman, C., Benz, W., Brack, A., Chazelas, B., Chelli, A., Cottin, H., Coudé du Foresto, V., Danchi, W., Defrère, D., den Herder, J.-W., Eiroa, C., Fridlund, M., Henning, Th., Johnston, K., Kaltenegger, L., Labadie, L., Lammer, H., Launhardt, R., Lawson, P., Lay, O.P., Liseau, R., Martin, S.R., Mawet, D., Mourard, D., Moutou, C., Mugnier, L., Paresce, F., Quirrenbach, A., Rabbia, Y., Rottgering, H.J.A., Rouan, D., Santos, N., Selsis, F., Serabyn, E., Westall, F., White, G., Ollivier, M., and Bordé, B. (2009) Darwin-an experimental astronomy mission to search for extrasolar planets. Experimental Astronomy 23:435-461.

Crawford, I. (1990) Interstellar travel: a review for astronomers. Quarterly Journal of the Royal Astronomical Society 31:377-400. Epicurus. (300 BCE) Letter to Herodotus.

Glassman, T., Newhart, L., Barber, G., Turnbull, M., and NWO study team. (2009) Planning an efficient search for extra-solar terrestrial planets: how to find exo-Earths with NWO [\#404.04]. In 213 ${ }^{\text {th }}$ Meeting of the American Astronomical Society, American Astronomical Society, Washington DC.
Guedes, J., Rivera, J., Davis, E., Laughlin, G., Quintana, E., and Fischer, D. (2008) Formation and detectability of terrestrial planets around Alpha Centauri B. Astrophys. J. 679:1582-1587. Kasper, M., Beuzit, J.-L., Vérinaud, Ch., Yaitskova, N., Baudoz, P., Boccaletti, A., Gratton, R.G., Hubin, N., Kerber, F., Roelfsema, R., Schmid, H.M., Thatte, N.A., Dohlen, K., Feldt, M., Venema, L., and Wolf, S. (2008) EPICS: the exoplanet imager for the E-ELT. Proc. Soc. Photo. Opt. Instrum. Eng. 7015:70151S70151S-12.

Kilston, S. (1999) The Project: an observatory/transport spaceship for discovering and populating habitable extrasolar terrestrial planets [\#97.09]. In 193 ${ }^{\text {rd }}$ Meeting of the American Astronomical Society, American Astronomical Society, Washington DC. Available online at http://homepage.mac .com/nancy_n_sven/Files/The-UP\%20Poster.pdf.

Koechlin, L., Serre, D., Debra, P., Pella, R., Peillon, Ch., Duchon, P., Gomez de Castro, A., Karovska, A., Desert, J.-M., Ehrenreich, D., Hebrard, G., Lecavelier des Etangs, A., Ferlet, R., Sing, D., and Vidal-Madjar, A. (2009) The Fresnel interferometric imager. Experimental Astronomy 23:379-402.

Labeyrie, A. (1996) Resolved imaging of extra-solar planets with future $10-100 \mathrm{~km}$ optical interferometric arrays. Astron. Astrophys. Supplement 118:517-524.

Labeyrie, A., Guillon, M., and Fournier, J.-M. (2005) Optics of laser trapped mirrors for large telescopes and hypertelescopes in space Proc. Soc. Photo. Opt. Instrum. Eng. 5899:307-316.

Lammer, H., Bredehöft, J.H., Coustenis, A., Khodachenko, M.L., Kaltenegger, L., Grasset, O., Prieur, D., Raulin, F., Ehrenfreund, P., Yamauchi, M., Wahlund, J.-E., Grießmeier, J.-M., Stangl, G., Cockell, C.S., Kulikov, Yu.N., Grenfell, J.L., and Rauer, H. (2009) What makes a planet habitable? Astronomy \& Astrophysics Review 17:181-249.

Lawson, P., Lay, O., Martin, S., Peters, R., Gappinger, R., Ksendzov, A., Scharf, D., Booth, A., Beichman, C., Serabyn, E., Johnston, K., and Danchi, W. (2008) Terrestrial Planet Finder interferometer: 2007-2008 progress and plans. Proc. Soc. Photo. Opt. Instrum. Eng. 7013:70132N-70132N-15.

Le Bris, K., Clement, J., and Strong, K. (2006) Measurement of the infrared cross sections and line broadening of CFC-113 and comparison with calculations [H51]. In $19^{\text {th }}$ International Conference on High Resolution Molecular Spectroscopy, Prague, Czech Republic, Aug. 29-Sept. 2, 2006. Available online at http://praha.uochb.cas.cz/praha06/BoA/eas/H51.gif.

Lillie, C. and TPF Phase 2 Study Contributors. (2001) TRW TPF architecture phase 1 study. Phase 2 final report. Available online at http://planetquest.jpl.nasa.gov/TPF/TPFrevue/ FinlReps/Trw/TRW12Fnl.pdf.

Mayor, M., Udry, S., Lovis, C., Pepe, F., Queloz, D., Benz, W., Bertaux, J.-L., Bouchy, F., Mordasini, C., and Segransan, D. (2009) The HARPS search for southern extra-solar planets. XIII. A planetary system with 3 Super-Earths $(4.2,6.9$, and 9.2 $\left.M_{\oplus}\right)$. Astron. Astrophys. 493:639-644.

Schneider, J. (2000) Extrasolar planets transits: detection and follow-up. In Proceedings of the VLT Opening Symposium, edited by J. Bergeron, A. Renzini, B. Fort, P. Rosati, R. Kudritzki, L. Pasquini, M. Mayor, and F. Paresce, Springer, Berlin, pp 499-504.

Schneider, J., Boccaletti, A., Mawet, D., Baudoz, P., Beuzit, J.-L., Doyon, R., Marley, M., Stam, D., Tinetti, G., Traub, W., Trauger, J., Aylward, A., Cho, J.Y-K., Keller, C.-U., Udry, S., and the SEE-COAST TEAM. (2009) The Super Earth Explorer: a coronagraphic off-axis space telescope. Experimental Astronomy 23:357-377.

Semyonov, O. (2009) Radiation hazard of relativistic interstellar flight. Acta Astronaut. 64:644-653. 
Shaklan, S. and Levine, M. (2008) Terrestrial Planet Finder Coronagraph mission overview. In Proceedings of the Conference In the Spirit of Bernard Lyot: The Direct Detection of Planets and Circumstellar Disks in the $21^{\text {st }}$ Century, edited by Paul Kalas, University of California, Berkeley, CA.

Stam, D. (2008) Spectropolarimetric signatures of Earth-like extrasolar planets. Astron. Astrophys. 482:989-1007.

Williams, D. and Gaidos, E. (2008) Detecting the glint of starlight on the oceans of distant planets. Icarus 195:927-937.
Address correspondence to: Jean Schneider

Observatoire de Paris-Meudon

LUTH

Meudon

France

E-mail: Jean.Schneider@obspm.fr 